\title{
LOS DEFECTOS ÓPTICOS DE LA VISIÓN EXPLICADOS POR ARISTÓTELES
}

\author{
Sergio Barbero Briones \\ Instituto de Óptica (CSIC) \\ sergio.barbero@csic.es
}

Recibido: 5 enero 2012; Aceptado: 13 agosto 2012

Cómo citar este artículo/ Citation: Barbero, Sergio (2013), “Los defectos ópticos de la visión explicados por Aristóteles”, Asclepio 65 (1): p005. doi: http://dx.doi.org/10.3989/asclepio.2013.05

RESUMEN: Aristóteles fue el primer autor griego en tratar de explicar los defectos ópticos de la visión. Dentro del marco de su teoría de la visión, y con la ayuda de múltiples observaciones empíricas de la naturaleza, intentó fundamentar las razones por las que las agudezas visuales difieren de unos sujetos a otros, así como la presencia de ciertas ametropías, discerniendo entre la miopía y la presbicia. Para Aristóteles la miopía, falta de agudeza de visión lejana, es debida a una proporción inadecuada de humedad en el ojo o a una morfología ocular anómala.

PALABRAS CLAVE: Visión; errores refractivos; óptica; miopía; Aristóteles.

\section{THE VISUAL REFRACTIVE ERRORS AS EXPLAINED BY ARISTOTLE}

ABSTRACT: Aristotle was the first Greek author trying to give an explanation of the optical defects of vision. Within the framework of his theory of vision and with the help of numerous empirical observations of natural phenomena, he tried to explain why visual acuity is different from one subject to another and the existence of ametropias, differentiating between myopia and presbyopia. In Aristotle, myopia, low visual acuity for far vision, is due to inappropriate humidity content inside the eye or because of anomalous eye morphology.

KEY WORDS: Vision; refractive errors; optics; myopia; Aristotle.

Copyright: () 2013 CSIC. Este es un artículo de acceso abierto distribuido bajo los términos de la licencia Creative Commons Attribution-Non Commercial (by-nc) Spain 3.0. 


\section{INTRODUCCIÓN}

El ojo humano actúa como un sistema óptico refractivo, cuya finalidad es formar imágenes en la retina -membrana interior del ojo responsable de recibir el estímulo visual- que reproduzcan, con la mayor fidelidad, los objetos vistos. Cuando la luz incidente en la retina no se focaliza formando una imagen con calidad aceptable se dice que el ojo adolece de errores refractivos o ametropías. Para visión lejana (sin esfuerzo acomodativo, relajado) dos son las principales ametropías: la miopía y la hipermetropía. En la primera, la luz se focaliza detrás de la retina, mientras que en la segunda, la imagen se forma delante.

El sistema visual humano posee la capacidad de actuar como un sistema óptico dinámico. La potencia refractiva del ojo, y por ende la capacidad de ver de lejos y de cerca, puede modificarse mediante cambios en la forma del cristalino. Es lo que se denomina: mecanismo de la acomodación. La presbicia es un defecto de la visión, asociado a la edad, provocado por la pérdida de la capacidad acomodativa, por lo que la visión de objetos cercanos se ve impedida.

En conjunto, los errores refractivos y la presbicia son los defectos ópticos de la visión más usuales, por lo que han sido siempre muy importantes en la oftalmología y optometría. A pesar de su trascendencia, los defectos ópticos de la visión no han sido estudiados con profusión en la historiografía del mundo clásico. Buena causa de esto es el hecho de que carezcamos de suficientes referencias alusivas en los textos clásicos griegos y romanos. Los escritos Aristotélicos (o pseudo-Aristotélicos) se encuentran dentro de los pocos donde se mencionan este tipo de defectos de forma implícita, además de explícitamente en los libros de Problemas ${ }^{1}$ (Aristóteles, 2004). De hecho, no parece que se hayan encontrado referencias a los errores refractivos previas a Aristóteles, incluso en textos médicos donde se analizan diversas enfermedades visuales, tales como los tratados Hipocráticos. Aunque, como bien advierte el historiador Julius Hirschberg (Hirschberg, 1982, p. 106), de esta omisión no se puede deducir que tales defectos no fuesen conocidos. De hecho, lo más probable, por su fácil observación, es que, por lo menos, fuesen reconocidos.

Según sostiene Julius Hirschberg (Hirschberg, 1982, p. 107), la primera vez que la palabra miope - del griego $\mu u \dot{\omega} \omega$ y de aquí myops en latín- aparece escrita en la literatura es en Problemas ${ }^{2}$. Myops $(\mu \dot{\omega} \omega \psi)$ es la composición de dos términos: myein, que significa cerrar, y ops $(\omega \psi)$ cuyo significado es ojo. Miope es, pues, aquel que contrae la pupila para ver mejor. Es lo que en la práctica optométrica se conoce como efecto estenopeico, del cual hablaremos más tarde. Curiosamente -o no tanto, considerando la mala visión de lejos de los insectos- myops era también en griego la palabra para denominar al tábano (Hirschberg, 1982, p. 107)

También en Problemas aparece la palabra présbita ( $\pi \rho \varepsilon \sigma B u ́ \tau \eta \varsigma)$, que en griego significaba hombre anciano, dándose aquí el significado más restringido de hombres ancianos con baja visión (Prob. 25 Sec. 31, 959b 38): “¿Por qué, padeciendo cierta debilidad de los ojos tanto el miope como el anciano ( $\pi \rho \varepsilon \sigma B u ́ \tau \eta \varsigma)$, uno acerca las cosas, si quiere ver, y el otro las aleja?".

Es oportuno, pues, preguntarse por las ideas que Aristóteles tenía acerca de los errores refractivos: de su diferenciación, desde el punto de vista conceptual, de su etiología y de su relación con su bien estudiada teoría de la visión. Conviene empezar repasando los supuestos de la teoría de la visión Aristotélica, previo brevísimo sumario de las teorías visuales pre-Aristotélicas.

\section{LAS TEORÍAS DE LA VISIÓN PRE-ARISTOTÉLICAS}

En la Antigüedad, la visión, de la misma manera que otros aspectos de la filosofía natural, fue objeto de múltiples y variadas teorías explicativas. Dentro de esta diversidad cabe destacar dos concepciones previas a Aristóteles.

La primera de ellas se debe a los llamados atomistas, aún reconociendo la diversidad de opiniones de los pensadores adscritos a esta escuela (Lindberg, 1976, pp. 2-3). En coherencia con su concepción eminentemente materialista, mantenían que la visión se produce por un contacto directo del órgano de la visión con cierto tipo de materia que emana de los objetos visibles en dirección a los ojos. Esta materia proveniente de los objetos - denominada eidola por Demócrito (c. 160 a. C.), o posteriormente simulacra por Lucrecio (c. 99 a. C. - c. 55 a.C.) - actúa sobre el órgano visual de manera análoga a como los objetos estimulan el sentido del tacto cuando los tocamos. La visión es, por tanto, un fenómeno netamente físico.

Aristóteles criticó duramente esta teoría mediante dos razonamientos. En primer lugar, refuta la reducción que hace Demócrito a "tangible todo lo sensible" (Acerca de la sensación y de lo sensible V, 442b 2). Aristóteles diferencia entre los objetos sensibles propios - de cada sentido-y los comunes ${ }^{3}$. No es posible, según el estagirita, reducir el objeto sensible propio de la visión (el color) a objeto sensible por cualquier otro sentido. En segundo lugar, esgrime un argumento mucho más simple e intuitivo. Si la visión es sólo debida a un fenómeno físico: "reflexión de una imagen" (Acerca de la sensación y de lo sensible II, 438a 9-10), ¿por qué no existen otros objetos aparte del ojo que puedan ver? Si la visión es un puro fenómeno físico, cualquier objeto que recibiese luz reflejada podría ver (Acerca de la sensación y de lo sensible II, 438a 11-14). La visión, pues, no puede ser únicamente algo físico- 
material. La percepción, como fenómeno psíquico, debe también contribuir al proceso visual.

Una segunda noción pre-Aristotélica establece que la visión, para que se produzca, necesita de la emanación de una especie de "fuego visual" proveniente del ojo. Esta idea, contrasta respecto de la teoría atomista, en el sentido de que se le da un mayor protagonismo al órgano sensorial ${ }^{4}$. La teoría visual Platónica, expuesta en el Timeo (45b-47c), es la más elaborada de todas las basadas en la noción del fuego visual. En la teoría platónica, la visión se produce por la coalescencia del fuego visual proveniente del ojo con la luz del sol, formando un cuerpo único y homogéneo. Empédocles (c. 493 a.C - c. 433 a.C), a pesar de la difícil interpretación de sus textos (Lindberg 1976 pp. 4-5), es visto por Aristóteles cómo defensor de la idea del fuego visual. Ésta es fácilmente rebatida por Aristóteles mediante un argumento de reducción al absurdo (Acerca de la sensación y de lo sensible II, 437b 12-17):

Si la vista fuera fuego, como dice Empédocles y como está escrito en el Timeo, y si la visión se produjera al salir una luz del ojo como de una linterna, ¿’por qué la vista no puede ver también en la oscuridad? ${ }^{5}$

\section{LA TEORÍA ARISTOTÉLICA DE LA VISIÓN}

Para Aristóteles, la sensación determina la generación del conocimiento -en franca oposición con la teoría de las ideas de su maestro-. De ahí, que el estudio de los sentidos y sensaciones sea primordial en su obra. Dos de sus más importantes tratados abordan esta temática: Acerca del alma y Acerca de la sensación y de lo sensible.

La entidad crucial en la teoría Aristotélica de las sensaciones es el alma. Así, fin fundamental de Acerca del alma es responder a la cuestión de qué es el alma (psyche). En el esquema Aristotélico de potencia-acto el alma es el acto primero de un ser viviente que posee la vida en potencia. El alma diferencia claramente a los seres vivientes de los no-vivientes, por lo que el estudio del alma es también el estudio de los seres vivientes y por ende de sus funciones vitales, dentro de las cuales Aristóteles asigna una especial importancia a la función sensorial.

En la teoría Aristotélica se distinguen tres agentes: el objeto sensible, el medio y el agente sensorial (Cappeletti, 1977, p.41). Para explicar el medio y el agente sensorial Aristóteles hace uso de la teoría presocrática (Empédocles) de los cuatro elementos - válida para Aristóteles en el mundo sublunar - constituyentes de la materia: aire, agua, tierra y fuego; y de las propiedades contrapuestas: caliente versus frío y húmedo versus seco. Dentro de las sensaciones, la vista, el oído y el olfato son sensaciones que son percibidas a través de un medio (principalmente agua o aire), es- tando los órganos sensoriales compuestos de los mismos elementos, ya que son su continuación.

Aristóteles rechaza tanto la teoría Platónica inmaterial de las sensaciones como la materialista de los atomistas. Con todo, la sensación no es algo únicamente inherente al cuerpo o al alma sino a ambos actuando de manera combinada 6 .

En Acerca del alma (II 6, 418a 28) se define, en primer lugar, el objeto sensible de la visión: "Lo visible, pues, es el objeto de la vista. Visible es, a su vez, el color"; aunque Aristóteles menciona otras tres clases de objetos visibles: los objetos fosforescentes, el fuego y el sol. Ahora bien, lo visible, el color ${ }^{7}$, lo es por el atributo de transparencia del medio existente entre el órgano sensorial de la vista, el ojo, y el objeto visible. La luz no es otra cosa sino la realización en acto de la transparencia.

Cuerpos transparentes, son esencialmente el agua y el aire, aunque existen otros. La transparencia esta además asociada al medio, de manera que en ausencia de medio (vacio) no habría transparencia y, en consecuencia, visión (Acerca del alma II 7, 419a 16).

No se expresa acertadamente Demócrito en este punto cuando opina que si se produjera el vacio entre el órgano y el objeto, se vería hasta el más mínimo detalle, hasta una hormiga que estuviera en el cielo. Esto es, desde luego, imposible. En efecto, la visión se produce cuando el órgano sensorial padece una cierta afección; ahora bien, es imposible que padezca influjo alguno bajo la acción del color percibido, luego a de ser bajo la acción de un agente intermedio; por fuerza ha de haber, pues, algo intermedio y, por tanto, hecho el vacío, no sólo no se verá hasta el más mínimo detalle, sino que no se verá en absoluto.

Descrito el objeto sensible (color) y el medio necesario (transparencia), queda por describir el órgano sensorial, el ojo. Aristóteles coincide con Demócrito al decir que el ojo está compuesto de agua -Platón y Empédocles creían que estaba compuesto de fuego- ya que el ojo debe ser continuación de lo transparente. Ahora bien, Aristóteles todavía se plantea la siguiente cuestión en su tratado: Partes de los animales (II 656b 1-4):

La vista, en todos los animales que la poseen, está lógicamente en torno al cerebro; pues el cerebro es húmedo y frío, y la vista es por naturaleza acuosa: el agua, entre las sustancias transparentes, es la más fácil de conservar guardada. ${ }^{8}$

En efecto, si el aire es más transparente que el agua ¿por qué el ojo es de naturaleza acuosa? - no hay que olvidar que una de las máximas más repetidas de Aristóteles es que la naturaleza no hace nada en vano-. La razón radica en que el aire por su naturaleza vo- 
látil no es tan "fácil de conservar guardada" como el agua. Esta última idea es especialmente relevante, ya que nos indica que la transparencia del ojo se mantiene mientras que éste conserve la cantidad de agua adecuada, la humedad necesaria. La humedad del ojo aparece como un factor determinante en la correcta composición del ojo.

\section{SOBRE LA CAPACIDAD VISUAL Y LOS DISTINTOS GRA- DOS DE VISIÓN}

Los escritos sobre biología suponen en torno a un tercio del total del Corpus Aristotelicum, siendo los más relevantes los tratados de zoología. Los que contienen información relevante para este estudio son: Investigación de los animales, Partes de los animales y Reproducción de de los animales.

El tratado Investigación de los animales, primero y más extenso de todos en el ámbito de la zoología, tiene un carácter expositivo. En el libro IX de este tratado (620a 1-6) Aristóteles recoge esta curiosa observación:

El quebrantahuesos tiene un leucoma en los ojos y su vista no es normal. En cambio el águila de mar tiene una vista penetrante y obliga a sus pequeños, cuando todavía están implumes, a mirar al sol a la cara, y al que no quiere lo golpea y lo gira hacia el astro; y al primero que le lloran los ojos lo mata, pero al otro lo cría.

Más allá de la credibilidad de lo descrito, esta observación es sumamente sugerente. Se dice que el águila "tiene una vista penetrante", esto es, una buena agudeza visual para visión lejana. A su vez, se menciona la extraña observación de que el águila obliga a sus crías a mirar al sol a la cara, lo cual implica mirar de lejos. Hoy en día sabemos que el crecimiento del ojo humano, como sistema óptico óptimo, se regula mediante un mecanismo de control homeostático (Wallman, 2004), denominado emetropización. Cuando el proceso de emetropización funciona incorrectamente, aparecen ametropías como la miopía o la hipermetropía. El desarrollo refractivo anómalo (ametropías) del ojo humano depende de factores genéticos y ambientales. Entre los factores ambientales, los determinados por estímulos visuales son fundamentales en el desarrollo refractivo, tal como han evidenciado numerosos experimentos con animales vertebrados (Sivak, 2008). Entre estos factores, el exceso de tareas de visión cercana -en edades tempranas- en detrimento de tareas de visión lejana se reconoce como un posible factor de riesgo en el desarrollo de la miopía (Goss, 2000). Aristóteles no parece establecer un efecto de causalidad entre ambas observaciones, a saber: el águila tiene buena vista debido a que la madre obliga a las crías a realizar tareas de visión lejana. Sin embargo las dos observaciones se dan conjuntas, lo cual no deja de ser altamente llamativo.
Por el momento, lo que Aristóteles deja claro, es que hay ciertos animales que tienen mejor vista de lo normal. La mera observación nos permite constatar que existen distintas capacidades visuales. Esto mismo ocurre entre personas. En Acerca de la generación y la corrupción (I, 328a 14-20) Aristóteles describe la diferencia entre un hombre que no posee vista aguda y otro que sí:

Si por el contrario, la combinación fuera una composición de pequeñas partículas, no ocurriría nada de esto, sino que los ingredientes sólo estarán combinados desde la perspectiva de la percepción visible, y el mismo objeto que a un hombre, si no posee vista aguda, le parece "combinado", no estará combinado a los ojo de Linceo.

La persona de vista aguda es Linceo $^{9}$, personaje de la mitología griega cuyo atributo más característico era su penetrante agudeza visual ${ }^{10}$.

El concepto de vista aguda queda explícitamente definido (Reproducción de los animales V, 780b 1619) como la capacidad de ver de lejos y discernir con resolución las diferencias entre los objetos vistos; definición ésta que, en esencia, es idéntica al concepto moderno de agudeza visual, que es la métrica de calidad visual más usada en la actualidad.

En el libro $X$ de Investigación de los animales se discute la esterilidad usando la siguiente analogía (633b 26-29):

Se dice también que la matriz que no está en buen estado es, sin embargo, capaz de ejercer como conviene y sin dolor su función propia, si su deterioro no afecta a la parte necesaria al ejercicio de la función. Así nada impide a la vista conservar su facultad de ver claramente, incluso si todas las partes del órgano no están en buen estado o presenta algún tumor.

Esta última frase es de suma importancia para entender cómo interpretaba Aristóteles los defectos ópticos de la visión. Aristóteles desliga la patología ocular de la propia capacidad de ver bien. Lo plantea más explícitamente en Reproducción de los animales (V, 780b 29-30): "La causa de que la vista sea tan aguda que distinga las diferencias, está en el ojo mismo". Lo errores refractivos no serían así fruto de una enfermedad curable ${ }^{11}$. Para Aristóteles, la agudeza visual tiene que ver más bien con la morfología ocular. Así los defectos visuales son debidos a una morfología ocular anormal. Dos características morfológicas son las que condicionan la agudeza visual: el grado de humedad - manifestado en el color de las pupilas - y la profundidad de los ojos respecto de la frente.

\section{LAS CAUSAS DE LAS DISTINTAS AGUDEZAS VISUALES}

El color de la pupila está ligado al grado de humedad del ojo (Reproducción de los animales V, 779b 
11): "los ojos que tienen mucha humedad son negros porque una cantidad grande no es transparente; y son azules los que tienen poca humedad". Los ojos claros son indicativos de menor humedad que los oscuros. Una humedad baja conlleva debilidad visual ${ }^{12}$, ya que, como hemos visto, el agua es el medio por el cual se manifiesta la transparencia del ojo. De esta manera el color azul es síntoma de debilidad visual ${ }^{13}$. Es fácil de percibir el hecho de que los recién nacidos ven mal de lejos, siendo el proceso de emetropización el mecanismo asociado con el crecimiento que permite corregir esta ametropía de los recién nacidos. Aristóteles asociaba esta debilidad visual de los recién nacidos a la falta de humedad en sus ojos - ojos azulados de los neonatos ${ }^{14}$ -

Ya que el hombre es (Investigación de los animales I, 492a 5): "el único, o casi el único de los animales cuyos ojos presentan colores variados" 15 , es posible la existencia de distintos grados de humedad en los ojos de distintos sujetos, y de aquí la presencia de distintos grados de agudeza visual entre hombres.

En cuanto a la morfología del ojo, en Investigación de los animales se afirma que los ojos más hundidos son los que mejor agudeza visual disponen, aunque a continuación se matiza esta aseveración, con su principio del término medio (Investigación de los animales I, 492a7-10):

Los ojos son grandes, pequeños o medianos. Estos son los mejores. Además, los hay salidos, hundidos o en posición intermedia. En toda clase de animales, cuanto más hundidos, más penetrantes son, pero la posición intermedia es señal de carácter excelente.

En obras ulteriores se manifestará más radical al respecto. Así en Reproducción de los animales (V, 780b 34- 781a 2):

Por otro lado, la causa de ver de lejos y de que llegue el movimiento procedente de los objetos visibles alejados está en la posición de los ojos: pues los animales con ojos prominentes no ven bien de lejos; en cambio, los que tienen los ojos metidos en una cavidad son capaces de ver de lejos porque el movimiento no se dispersa en el espacio sino que va directo.

Los ojos hundidos en la frente proporcionan una mejor agudeza visual porque conducen de mejor manera el movimiento que estimula la visión. En un caso ideal Aristóteles llega a imaginar que (Reproducción de los animales V, 781a 7-10): "De hecho, se verían especialmente bien las cosas distantes, si hubiera una especie de tubo continuo desde el ojo hasta el objeto contemplado" ${ }^{\prime 6}$. A continuación de este texto Aristóteles plantea una interesante discusión en la que afirma que esto es cierto independientemente de si se usase el concepto de "fuego visual" proveniente del ojo, ya que la visión depende del movimiento direc- cional, sea éste en un sentido (del objeto al ojo) o en el otro (del ojo al objeto).

El tratado Partes de los animales es probablemente posterior a Investigación de los animales. Mientras en este tratado se describían observaciones, en aquel se pretende explicar las causas de los fenómenos observados. Puede considerarse el primer estudio escrito en anatomía comparada, por lo este libro es de especial relevancia para entender por qué para Aristóteles unos animales tienen mejor agudeza visual que otros. A lo largo del texto se trata de explicar de que manera los animales cumplen sus fines determinados. La concepción teleológica de la naturaleza en Aristóteles es coherente con su teoría anatómica. La morfología y comportamiento de los órganos se encuentra adaptada a la función de estos en la vida de los animales, que difiere entre especies. En cuanto a los ojos (Partes de los animales II, 657b 24-30):

Los cuadrúpedos ovíparos no parpadean de la misma manera, porque, al vivir en tierra, no les es preciso tener el ojo húmedo ni la vista aguda. En cambio, a las aves les es necesario: desde lo lejos, en efecto, es su uso de la vista. Por eso, también las rapaces son muy agudas de vista (pues desde arriba avistan su alimento, y por ello también vuelan más alto que las otras aves), en tanto que las aves viven en tierra y no son voladoras, como los gallos y otros afines, no son agudas de vista, pues no les urge para su vida.

El grado de humedad es regulado por la acción de los párpados, órganos de piel dura. La finalidad de estos, según Aristóteles, es la de proteger la vista de agentes externos, librando de este cometido a lo que rodea a la pupila, la cual debe ser fina y acuosa para permitir la transparencia necesaria para la visión. EI mecanismo regulador es la intensidad de parpadeo, que modula el nivel de humedad y por tanto la agudeza de vista. Algunos animales, como los peces no poseen párpados, puesto que el agua no contiene tantos objetos peligrosos como el aire. En estos, la ausencia de párpados pretende compensar la menor transparencia del agua respecto del aire ${ }^{17}$.

\section{DEGRADACIÓN CON LA EDAD (PRESBICIA)}

Aristóteles afirma que el intelecto es de naturaleza incorruptible, y por tanto también la capacidad sensorial del alma. No es el alma lo que envejece, sino el cuerpo $^{18}$. El deterioro del órgano sensorial, del ojo, es por tanto lo que provoca la debilidad de la vista con la edad; y esto es así de manera que: "si un anciano pudiera disponer de un ojo apropiado vería sin duda, igual que un joven" (Acerca del alma I 4, 408b 22).

Por tanto, la presbicia - degradación de la vista con el envejecimiento- aparece por un cambio en la propia morfología del ojo. Pero: ¿de qué manera concreta se produce ésta? La vida se va degradando con la 
edad por medio de un movimiento de corrupción de lo caliente-húmedo a lo frío-seco ${ }^{19}$. Sin embargo, curiosamente, a pesar de que lo más lógico hubiese sido que Aristóteles hubiese explicado la presbicia por medio de los cambios de humedad con la edad, prefiere plantear una nueva e interesante causa morfológica (Reproducción de los animales V, 780a 27-35):

Las causas de una vista débil o aguda no son sólo las mencionadas, sino también la naturaleza de la piel que cubre lo que llamas la niña del ojo. Es necesario que esa piel sea transparente, y una piel así debe ser fina, blanca y lisa: fina para que el movimiento del exterior entre directo; lisa, para que no haga sombras con las arrugas (también por eso los viejos no tienen una vista aguda: pues igual que el resto de la piel, también la del ojo se arruga y se hace más espesa con la vejez); y blanca, porque lo negro no es transparente: de hecho en eso es en lo que consiste lo negro, en no ser transparente.

La explicación Aristotélica de la presbicia ${ }^{20}$ es que la piel que recubre la pupila se arruga con la edad, y esto hace que la sensación visual, a través del movimiento del exterior hacia el interior, produzca sombras.

\section{LOS ANÁLISIS GEOMÉTRICOS EN METEORÓLOGICOS $Y$ EN PROBLEMAS}

Meteorológicos es un conjunto de cuatro libros que tratan sobre lo "alto en el cielo", aunque en realidad abordan un amplio abanico de fenómenos, no sólo atmosféricos sino también hidrológicos, geofísicos, etc. La datación de estos libros Aristotélicos es controvertida, aunque la hipótesis más probable es que fuesen escritos antes de su obra zoológica ya mencionada. De hecho, una aparente evolución de la teoría de la visión Aristotélica en esta obra respecto de la expuesta en los tratados zoológicos refuerza esta hipótesis.

En el libro III se pretende interpretar una serie de fenómenos ópticos atmosféricos, tales como los halos y el arcoíris. Para esto, Aristóteles hace uso del concepto de la reflexión de la luz ${ }^{21}$, fenómeno bien conocido pero no muy bien comprendido en su época. De hecho, de sus escritos es difícil concluir si Aristóteles conocía el principio de ángulos iguales que rige la reflexión de la luz cuando incide sobre una superficie reflectora (Boyer, 1946); (Merker, 2002). Inherente a la noción de reflexión, Aristóteles tuvo que aplicar el concepto de rayo visual (ó $\psi \iota \varsigma, o p s i s$ ), que necesariamente tuvo que tomar prestado, quizás de fuentes Platónicas, ya que, como hemos visto, su propia teoría ignoraba tal concepto. Es, por tanto, manifiesta la inconsistencia entre el análisis geométrico, basado en la reflexión, de Meteorológicos y su teoría visual posterior. David Lindberg sugiere que lo más razonable es interpretarlo como una evolución del pensamiento de Aristóteles, adscribiendo las explicaciones de los
Meteorológicos a una etapa influida por el Platonismo (Lindberg, 1976, p. 218). Con todo es conveniente matizar, como apunta Beare (Beare, 1906, pp. 65-67), que el rayo visual en Meteorológica es una entidad usada per se, sin estar necesariamente asociada al objeto sensible $u$ al órgano sensorial (Meteorológicos III, 374b 24): "Y no hay diferencia entre que cambie lo visto o cambie la vista: pues en ambos casos será lo mismo"22.

El marco de análisis geométrico de Meteorológicos, conduce a una explicación diferente de los defectos visuales. En este contexto, la debilidad de la vista se relaciona con la presencia de la reflexión de la luz ${ }^{23}$. En Meteorológicos (Meteorológicos III, 466a 18-19): se describe el curioso caso del hombre - probablemente hace referencia a Antiferón de Oreo (Lehoux, 2007) - que veía con muy poca agudeza visual, porque su propia visión se reflejaba hacia él ya que el aire que se encontraba de frente reflectaba su visión "pues era tan débil y absolutamente tenue, por su <estado de> agotamiento" (Meteorológicos III, 373 a35-b10). De esta manera Aristóteles introduce una causa de debilidad visual que, al contrario de lo visto hasta ahora, no depende de una causa física, sino de algo dependiente de la capacidad sensitiva. Una obvia interpretación de esta explicación es que Aristóteles aceptase en ella la emisión de cierta sustancia asociada con el rayo visual ${ }^{24}$.

Problemas es una colección de problemas de diversa índole, planteados en forma de preguntas, a las que a continuación se trata de responder, mostrando una o varias respuestas. A lo largo de Problemas, se trata de dar explicación a ciertas observaciones sobre el fenómeno de la visión ${ }^{25}$. Lo paradójico de estas aclaraciones es que más que hacer uso de la teoría Aristotélica de la visión se utilizan conceptos, de manera análoga a lo que ocurre en Meteorológicos, más provenientes de un análisis geométrico de la visión. Las herramientas usadas son las de rayo visual y cono visual -las de la óptica Euclidiana (Burton, 1945)-. Una posible explicación de esta nueva contradicción es que las respuestas proporcionadas en Problemas fuesen, en estos casos, posteriores adaptaciones de la escuela peripatética a nuevos conceptos. Filius (Filius, 1997, pp. 77-83) ha comparado la traducción árabe efectuada en el siglo IX de Problemas con el texto original griego, encontrando que mientras en el manuscrito árabe se asume un modelo Galénico de rayos visuales saliendo del ojo, en el texto griego se sigue asumiendo un modelo geométrico, pero ahora, asumiendo rayos entrando dentro del ojo. De esta manera el texto griego es más fiel al espíritu Aristotélico. Con todo, es interesante señalar que el modelo de cono visual es común a lo explicado en Meteorológica.

Como botón de muestra en el problema 9 de la sección tercera se responde a la cuestión de por qué los 
borrachos son incapaces de ver las cosas lejanas (Problemas IX 3, 871b 35): "Pero (el rayo visualı se mueve en círculo por la constitución que tiene la visión: pues es un cono cuya base es un círculo".

\section{EL EFECTO ESTENOPEICO}

El efecto estenopeico es un fenómeno óptico (Keating, 2002, pp.23-24) basado en la reducción del tamaño de la mancha (spot) de luz generado por un objeto visto a través de un orificio. En el caso límite de un objeto puntual y de un tamaño de apertura infinitesimalmente pequeño, de manera que sólo dejase pasar un rayo, la mancha de luz sería un único punto con lo que existiría una correspondencia perfecta entre punto objeto e imagen, lo que se denomina un sistema óptico perfecto ${ }^{26}$.

El efecto estenopeico se ha utilizado, desde hace tiempo, cómo una técnica primaria de evaluación visual. Si la visión a través de un agujero, de un diámetro específico, mejora, puede significar que se tenga un defecto refractivo en la visión ${ }^{27}$.

Una de las grandes controversias en la historia de la óptica antigua es la hipótesis de si las civilizaciones antiguas usaban algún tipo de tecnología óptica para la compensación de los defectos ópticos de la visión ${ }^{28}$. La controversia viene justificada por la práctica ausencia de fuentes escritas que mencionen el uso de este tipo de tecnología. Tan sólo existen algunas referencias al efecto magnificador de objetos de vidrio rellenos de agua. La más conocida de ellas es la del cordobés Seneca (c. 4 a.C - c. 65 d.C). En Cuestiones Naturales (Lib. I, 6.5) escribe: "Los caracteres gráficos, aunque sean diminutos y confusos, se ven mayores y más claros a través de una esfera llena de agua" (Seneca, 1979, p.71). También se pueden encontrar referencias en los Saturnales de Macrobio (Temple, 2000, p.71).

Algunos autores mantienen que el uso de lentes estaba ampliamente extendido (Sines, 1987) y ponen como ejemplo la multitud de obras artísticas miniaturizadas, documentadas en registros arqueológicos, que difícilmente pudieron ser hechas bajo condiciones normales. Otros autores (Gorelick, 1981; Plantzos, 1997) sostienen que los mismos trabajos pudieron ser realizados por sujetos miopes - los miopes tienen mayor agudeza visual para visión cercana-. A colación de estas controversias surgió una sugerente hipótesis histórica, la cual plantea que el efecto estenopéico fue de facto una técnica de ayuda visual, por ejemplo utilizada por los artesanos que tallaban miniaturas (Wilk, 2006). Esta hipótesis encuentra cierta ayuda en los textos de Problemas. Ya vimos como la propia palabra miopía, describe la corrección estenopéica usada por los miopes. De hecho en los Problemas se da una explicación de la corrección estenopéica basada en la teoría de flujo de rayos visuales (Problemas, Prob. 8 Sec. 31, 958a 35):
¿Por qué los miopes escriben letras pequeñas?" Pues es extraño el hecho de que no teniendo una buena vista hagan un trabajo propio de gente con vista penetrante. ¿Acaso porque las cosas pequeñas parecen grandes si están cerca? Y estos escriben acercándose. ¿O es que acaso escriben contrayendo los párpados? Pues, por debilidad de la vista, si escriben con los ojos totalmente abiertos, la vista se dispersa y la visión es débil, pero del otro modo la visión llega de forma concentrada.

Los párpados se entornan para reducir el tamaño del cono visual, de manera que la superficie del objeto de la visión se reduce, y en consecuencia la visión se concentra más en esa área con lo que aumenta su resolución. Ésta es, de nuevo, una explicación más propia de la teoría geométrica de la visión. En la misma sección, en el problema 16, se vuelve a retomar el asunto (Problemas, Prob. $16 \mathrm{Sec} .31,959$ a 1-10):

¿Por qué los miopes ven contrayendo los párpados? ¿Es por debilidad de la vista? Igual que los que para ver de lejos aplican la mano, así también para ver de cerca colocan los párpados como la mano. Hacen esto para que la visión salga más concentrada, al salir a través de un espacio menor, y no se disperse inmediatamente por salir de un espacio muy abierto. Una mayor cantidad de visión mejora la vista.

Lo interesante aquí es la mención explícita de una acción consciente para mejorar la visión: aplicar la mano, de manera que el cono de luz se reduzca. Es razonable, pensar que de la misma manera se podía utilizar -o incluso construir - una especie de tubo tal como vimos que se teorizaba en Reproducción de los animales (cf. Reproducción de los animales V, 781a 7-10). De hecho, en este sentido, Robert Eisler (1949) sugirió que en la antigüedad se usaban tubos vacios para guiar la mirada en el firmamento, y por tanto ver mejor las estrellas.

\section{CONCLUSIONES}

Tras Aristóteles, en la antigüedad clásica, no se encuentran referencias escritas tratando de explicar los defectos ópticos de la visión, hasta que siglos después lo hagan Seneca, Plutarco (c. $46-120$ d. C.), Lucrecio o Ptolomeo (c. 100 d. C. - c 170 d. C.). De todas ellas, destacan las referencias del matemático y astrónomo Claudio Ptolomeo. Mark Smith ha analizado las fuentes de inspiración Aristotélicas en el trabajo de Ptolomeo (Smith 1988), que influyeron, no tanto en la teoría física de la visión Ptolemaica, sino sobre todo en la concepción físico-psicológica del proceso visual, con especial interés en la fase psicofísica de la percepción visual. En el libro segundo del manuscrito Ptolemaico de Óptica ${ }^{29}$ se lee lo siguiente:

Aquellos que tienen ojos profundos ven más lejos que aquellos que no tienen tales ojos; y la razón de 
esto es que la potencia visual de aquellos está comprimida, ya que al emanar a través de sitios estrechos, el flujo visual se comprime y alarga.

Ptolomeo, pues, comparte la idea Aristotélica de que cierta morfología de los ojos (cf. Investigación de los animales I, 492a7-10 y Partes de los animales II, 657b 24-30) hace que estos posean una mayor agudeza visual de lejos. La explicación es la misma que la expuesta en los Problemas (cf. Prob. 8 y $16 \mathrm{Sec}$. 31): una reducción del flujo visual, lo concentra y por tanto mejora la agudeza visual. Ptolomeo también utiliza el concepto Aristotélico de pérdida de humedad para explicar la falta de agudeza visual en visión cercana (Smith, 1996, p. 106). Vemos, pues, que los conceptos aristotélicos básicos para explicar los defectos ópticos de la visión fueron plenamente asumidos por Ptolomeo.

Tan pronto como el corpus Aristotélico, mediante las traducciones medievales, empezó a estar disponible, primero entre estudiosos del mundo árabe, y después dentro del ámbito cristiano, surgieron varios

\section{NOTAS}

1 Sobre la autoría, o no, Aristotélica de los Problemata sigue sin existir consenso.

2 “¿Por qué los miopes ( $\mu$ $\omega \pi \varepsilon \varsigma$ ) escriben letras pequeñas?" (Prob. 8 Sec. 31, 958a 35). “¿Por qué los miopes no tienen visión aguda, pero escriben pequeño?" (Prob. $15 \mathrm{Sec}$. 31, 958b 34). “¿Por qué los miopes ven contrayendo los párpados?" (Prob. 16 Sec. 31, 959 a 2).

3 En una pequeña digresión del capítulo $\mathrm{V}$ del libro $\mathrm{X}$ de la Moral a Nicómaco, Aristóteles ahonda en la diferencia entre tacto y vista, mostrando una preferencia clara por la vista: "Pero así como los actos de los sentidos son diferentes, también lo son sus placeres. La vista difiere del tacto por su pureza y exactitud".

4 Aunque pueda parecer una idea poco intuitiva o extraña, es de resaltar el hecho de que algunos estudios psicológicos modernos realizados en niños e incluso adultos, comenzando con ciertas observaciones de Jean Piaget, han puesto de manifiesto que ciertas creencias acerca del ojo como órgano activo emisor de la visión siguen estando presentes en la actualidad. (Winger, 2002).

5 Conviene señalar que Platón, consciente de este hecho obvio, explica que la visión no es posible durante la noche porque al faltar la luz diurna no es posible la unidad de fuegos necesaria para la visión Timeo (45d): "Durante la noche el fuego parecido se va, y el otro queda amputado; pues al venir junto a lo diferente, se transforma y se apaga al no formar ya una unidad con el aire que carece de fuego". pensadores que asumieron, aún con algún matiz, el marco Aristotélico de la naturaleza de la luz y la visión. No es de extrañar que los conceptos Aristotélicos de la visión fuesen un punto de referencia fundamental en cualquier discusión sobre la visión en buena parte de la Edad Media y principios de la Moderna. Hasta el punto de que Johannes Kepler (1571-1630) se vio impelido a incluir en su obra magna de óptica $\mathrm{Ad}$ Vitellionem Paralipomena (Kepler, 2000) un apéndice específico para rebatir alguno de los argumentos de la teoría de la visión de Aristóteles.

En otro tratado posterior (Kepler, 1611), Kepler reconoce el origen etimológicamente Aristotélico de los términos miopía y presbicia ${ }^{30}$. El hecho de que Kepler diese la primera explicación correcta, según el conocimiento moderno, de la miopía y la hipermetropía, y la influencia crucial de sus tratados de óptica en las concepciones de la óptica modernas, ayudaron, probablemente, a que estos términos Aristotélicos acabasen finalmente por imponerse en la ciencia óptica moderna.

6 Esto se ha sintetizado de la siguiente manera: "La sensación consiste en la recepción de las formas sensibles del objeto, desprovistas de su materia por el sensorio" (Cappeletti, 1977, p.17).

7 La teoría de los colores Aristotélica, establece que los colores son combinaciones de la mezcla de dos contrarios: luz-oscuridad, blanco-negro. De manera que los colores se producen en sucesión dependiendo de si la luz es más intensa - colores con más blanco como el rojo- o menos - colores con menos blanco como el violeta-.

8 Cf. Acerca de la sensación y de lo sensible (II, 438a 13-16): “Que el ojo es de agua es cierto, pero, sin embargo, no se produce la visión porque sea de agua, sino porque es transparente, lo cual es también común al aire, pero el agua es más fácil de contener y más densa".

9 Posible epónimo proveniente del nombre de animal: lince, del cual es sabido su gran agudeza visual. La expresión "tener vista de lince" tiene su probable origen en esta figura. Sobre la relación de este personaje de la mitología, el lince y la pionera sociedad científica Accademia dei Lincei ver Lüthy (1996).

10 Hijo de Arene y Afareo, de la estirpe de los Deucaliónides. Según la leyenda, Linceo, en compañía de su hermano Idas, se enfrentó a los Dioscuros en disputa por unas cabezas de ganado. Cuando los Dioscuros intentaron esconderse para tender una emboscada a Linceo, éste subió a una montaña del Taigeto y desde allí, gracias a su agudeza visual pudo descubrir a los Dioscuros escondidos dentro de un roble hueco (Cano, 2004, p. 680) 
11 En tratados bizantinos posteriores (e.g. los siete libros de Pablo de Egina) la miopía se caracterizó como una condición incurable (Magnus, 1999).

12 Aunque en el tratado Meteorológicos (374 a 24-30) se dice que en los ojos muy húmedos se puede producir un efecto de reflexión de la luz que debilite la vista. Lo singular de las explicaciones de Meteorológicos se expondrá más adelante.

13 En Investigación de los animales se menciona el amarillo como el color más favorable (I 492a 1-4): "El blanco de los ojos es generalmente igual en todos los animales, pero el llamado negro [la pupila] difiere. En unos es negro, en otros azul intenso, en otros gris, en algunos amarillento, como los ojos de la cabra: este color es señal de un carácter excelente y es el más favorable a la agudeza visual".

14 (Reproducción de los animales V, 779b 11) "Respecto a que los niños tengan los ojos azules y ningún otro color, la causa es que los órganos de los jóvenes son más débiles, y el color azul es un tipo de debilidad".

15 A excepción del caballo (Reproducción de los animales $\mathrm{V}, 779 \mathrm{~b}$ 4): "Pero entre los demás animales, es en particular el caballo el que presenta colores variados".

16 El uso de la palabra "tubo" (aulo) en este fragmento se ha querido interpretar por parte de Robert Temple (Temple, 2000, pp. 157-160) como una prueba de la utilización de telescopios en la civilización griega; afirmación a mi parecer poco argumentada. Es mucho más probable que Aristóteles tuviese en mente el hecho de que en la antigüedad probablemente se usaban tubos vacios para observar el firmamento (Eisler, 1949).

17 "Los peces tienen ojos húmedos" (Partes de los animales II, 658a4).

18 "De manera que la vejez no consiste en que el alma sufra desperfecto alguno, sino en que lo sufra el cuerpo en que se encuentra" (Acerca del alma I 4, 408b 24).

19 Tal teoría aparece claramente expuesta en la sección quinta del breve tratado Aristotélico: Acerca de la longevidad y de la brevedad de la vida.

20 Conviene advertir que, quizás aquí, Aristóteles en vez de explicar la presbicia, estuviese intentando dar una explicación de la aparición de las cataratas. Probablemente ambas patologías, cataratas y presbicia, se confundían.

21 Reflexión y refracción ópticas se confunden en Aristóteles. De

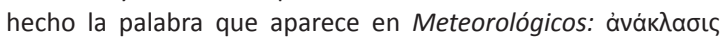
(anaklasis) se ha llegado a traducir en alguna ocasión como refracción. Ver (Boyer, 1946; Merker, 2000).

22 El mismo argumento se usa en Problemas (Problema IX, sección III): "De hecho, no hay ninguna diferencia entre que se mueva la vista o el objeto visto".

23 Como la vista del color, que es lo visible, depende de la transparencia (cantidad de luz), los diferentes colores dependen de la "debilidad de la vista". Incluso puede devenir en una percepción errónea del color (Meteorológicos III, 372b 7): "Ahora bien, el color de las cosas brillantes parece a veces brillante, pero otras veces, bien por estar empañado el espejo, bien por la debilidad de la vista, produce la apariencia de otro color".

24 Por ejemplo el pneuma de los estoicos. Esta interpretación se refuerza en el siguiente fragmento (Meteorológicos III, 374b 13): «la vista, al extenderse, se vuelve cada vez más débil y escasa».

25 Ya se ha mencionado que en Problemas aparece por primera

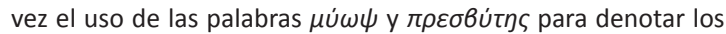
defectos visuales de vista lejana y cercana respectivamente.

26 Conviene matizar que esto sólo es válido dentro de la teoría geométrica de la luz; por debajo de un tamaño de apertura determinado los efectos difractivos hacen que la mancha aumente.

27 Es interesante remarcar que los Nautilius poseen una visión basada en el efecto estenopeico: Ver (Land, 2002, pp.57-58).

28 Para una introducción a la controversia ver Enoch (2007) e Ilardi (2007).

29 Traducido de la edición inglesa de Smith (1996, p. 107)

30 KEPLER, J. (1611), p. 16. Propositio LXIV: «Sunt qui remonta distinctè vident, propinqua confusè, quos Aristoteles appellat $\pi \rho \varepsilon \sigma B u ́ \pi \eta \varsigma$ : funt qui propinqua distinct, remota confuse, qui

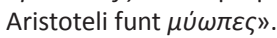




\section{BIBLIOGRAFÍA}

Aristóteles (2004), Problemas, Madrid, Editorial Gredos.

Aristóteles (1987), Acerca de la generación y la corrupción. Tratados breves de historia natural, Madrid, Editorial Gredos.

Aristóteles (1995), Moral a Nicómaco, Madrid, Espasa Calpe.

Aristóteles (1983), Acerca del alma, Madrid, Editorial Gredos.

Aristóteles (2000), Partes de los animales; Marcha de los animales; Movimiento de los animales, Madrid, Editorial Gredos.

Aristóteles (1992), Investigación sobre animales, Madrid, Editorial Gredos.

Aristóteles (1994), Reproducción de los animales, Madrid, Editorial Gredos.

Aristóteles (1996), Acerca del cielo; Meteorológicos, Madrid, Editorial Gredos.

Beare, J. (1906), Greek theories of elementary cognition from Alcmaeon to Aristotle, Oxford, Clarendon Press.

Boyer, C. (1946), "Aristotelian References to the Law of Reflection". Isis, 36(2), 92-95.

Burton, E.H. (1945), "The optics of Euclid", Journal of Optical Society of America, 35(5), 357-372.

Cano, J. (2004), El gran libro de la mitología griega. Madrid, La esfera de los libros.

Cappeletti, A.J. (1977), La teoría aristotélica de la visión, Caracas, Sociedad Venezolana de Ciencias Humanas.

Enoch, J. M. (1998), "The cover design: The enigma of early lens use", Technology and Culture, 39 (2), 273-291.

Eisler, R. (1949), "The polar sighting tube", Archives internationales d'historie des sciences, 2, 312-332.

Filius, L. S. (1997), "The theory of vision in the Problemata physica. A comparison between the Greek and Arabic versions". En: Endress, G. (coord.), The ancient tradition in Christian and Islamic Hellenism, Leiden, Research School Cnws.

Gorelick, L. Y. y Gwinnett, A. J. (1981), “Close work without magnifying lenses?", Expedition, 23 (2), 27-34.

Goss, D. A. (2000), “Nearwork and myopia”, Lancet, 356, 1456-1457.

Hirschberg, J. (1982), The history of ophthalmology. Volume 1, Bonn, Wayenborgh.

Ilardi, V. (2007), Renaissance vision from spectacles to telescopes, Philadelphia, American Philosophical Society.

Keating, M. P. (2002), Geometric, physical, and visual optics, Boston, Butterworth-Heinemann.
Kepler, J. (2000), Optics: Paralipomena to Witelo \& optical part of astronomy, Santa Fe, Green Lion Press.

Kepler, J. (1611), Facsímil (2009), Dioptrice seu Demonstratio eorum quæ visui et visibilibus propter conspicilla non ita pridem inventa accidunt, Kessinger Publishing.

Land, M. F. and Nilsson, D. (2002), Animal eyes, New York, Oxford University Press.

Lehoux, D. (2007), "Observers, Objects, and the Embedded Eye; or, Seeing and Knowing in Ptolemy and Galen", Isis, 98 (3), 447-467.

Lindberg, D. (1976), Theories of vision from al-Kindi to Kepler, Chicago, Chicago Press.

Lüthy, C. H. (1996), "Atomism, lynceus, and the fate of seventeenthcentury microscopy", Early Science and Medicine, 1 (1), 1-27.

Magnus, H. (1999), Ophthalmology of the ancients: Part 2, Oostende, Wayenborgh.

Merker, A. (2002),"Aristotle and the rainbow: Philosophical issues and scientific study". Archives for History of Exact Sciences, 56 (3), 183-238.

Plantzos, D. (1997), "Crystals and lenses in the Graeco-Roman world", American Journal of Archaeology, 101 (3), 451-464.

Platón (2003), lón, Timeo, Critias, Madrid, Alianza.

Séneca (1979), Cuestiones naturales, Salamanca, CSIC

Sines, G. y Sakellarakis, Y. A. (1987), "Lenses in antiquity", American Journal of Archaeology, 91 (2), 191-196.

Sivak, J. G. (2008), "The role of the lens in refractive development of the eye: Animal models of ametropia", Experimental Eye Research, 87, 3-8.

Smith, A. M. (1996), Ptolemy's theory of visual perception: an English translation of the Optics, Philadelphia, American Philosophical Society.

Smith, A. M. (1988), "The psychology of visual perception in Ptolemy optics", Isis, 79, 189-207.

Temple, R. (2000), El sol de cristal: tecnologías perdidas de la antigüedad, Madrid, Oberón.

Wallman, J. y Winawer, J. (2004), "Homeostasis of eye growth and the question of myopia". Neuron, 43, 447-468.

Wilk, S. R. (2006), "Ancient Optics: Producing magnification without lenses". Optics Photonic News, April, 1-13.

Winer, G. A. (2002), "Fundamentally misunderstanding visual perception: Adults' belief in visual emissions", American Psychologist, 57 (7), 417-424. 